\title{
USE OF A LOW-COST TEMPLATE-FREE ZSM-5 FOR ATMOSPHERIC PETROLEUM RESIDUE PYROLYSIS
}

\author{
Vinícius P. S. Caldeira ${ }^{a, *}$, Anne G. D. Santos ${ }^{a}$, Sibele B. C. Pergher ${ }^{b}$, Maria J. F. Costa ${ }^{b}$ and Antonio S. Araujo \\ a Departamento de Química, Universidade do Estado do Rio Grande do Norte, 59610-210, Mossoró - RN, Brasil \\ 'Instituto de Química, Universidade Federal do Rio Grande do Norte, 59054-180, Natal - RN, Brasil
}

Recebido em 10/09/2015; aceito em 19/11/2015; publicado na web em 17/02/2016

\begin{abstract}
To understand the physicochemical properties and catalytic activity during the pyrolysis of atmospheric petroleum residue, a templatefree ZSM-5 zeolite was synthesized using a direct method without additional seeds or an organic structure director and compared with conventionally synthesized ZSM-5. The crystallinities of the two zeolites were evaluated by XRD and FTIR and were quite similar; however, structural analyses using SEM and argon physisorption revealed that the zeolites diverged in particle diameter and in the external surface area of the micropores. The synthesis procedure without a template incorporated additional aluminum into the crystalline network, according to ICP-AES and TPD $\mathrm{NH}_{3}$ experiments. The catalytic pyrolysis performed over the template-free ZSM-5 generated results comparable to those for pyrolysis performed over the conventional ZSM-5 according to its hydrocarbon distribution. The selectivity to aromatics compounds was exactly the same for both ZSM-5 zeolites, and these values stand out compared to thermal pyrolysis. The template-free ZSM-5 produced $20 \%$ of light hydrocarbons $\left(\mathrm{C}_{4}-\mathrm{C}_{6}\right)$, where such compounds are olefins and paraffins of great interest to the petrochemical industry. Therefore, template-free ZSM- 5 is promising for industrial use due to its lowered synthesis time, low-cost and significant distribution to light hydrocarbons.
\end{abstract}

Keywords: template-free; zeolite; pyrolysis; petroleum residue.

\section{INTRODUCTION}

A zeolite is a crystalline aluminosilicate; the structure of these materials involves a combined tetrahedra network that generates channels as cavities or enclosures. Therefore, these materials have specific properties, including ion exchange and reversible desorption of water. ${ }^{1,2}$ This intra-crystalline space contains enclosures and channels and enables mass transfer that is limited by the pore diameter; these qualities impart shape selectivity for reagents, intermediates molecules and products. This shape selectivity is one of the most important properties of zeolites, which can also be called molecular sieves. ${ }^{1}$ ZSM-5 is a medium-pore ( $\sim 6 \AA$ ) zeolite with three-dimensional channels. This system of straight and sinusoidal channels that intersects, as defined by its 10-membered rings. A significant amount of research describing this highly siliceous pentasil zeolite has been conducted due to its unique channel structure, thermal stability, acidity and shape-selective properties. ${ }^{3-5}$

Since their introduction in refining processes, zeolites have been widely used during petroleum refining, as well as in the petrochemical industries, for selective adsorption and acid-base catalysis. ${ }^{6} \mathrm{ZSM}-5$ has been used to catalyze various hydrocarbon transformation reactions. Specially, ZSM-5 acts as a separative additive catalyst during the fluid catalytic cracking (FCC) process; this material can isomerize xylenes via disproportionation to form toluene and trimethylbenzene, while olefins can be aromatized over H-ZSM-5 (protonic form of ZSM-5, which provides greater acidity) at atmospheric pressure. ${ }^{3,7}$

Using organic templates when synthesizing zeolites can cause problems, including high production costs, poisoning, air pollution and coke deposition on the catalyst after incomplete decomposition of the organic material. Consequently, many researchers have studied the synthesis of ZSM-5 without using templates, obtainig satisfactory results by varying the $\mathrm{Si} / \mathrm{Al}$ ratio, ${ }^{7}$ utilizing a hydrothermal synthesis with agitation, ${ }^{8,9}$ comparing the syntheses under various templates, ${ }^{4}$ using a two-step process that separates the nucleation step for

*e-mail: vinicius_psc@yahoo.com.br crystallization, ${ }^{3}$ using sulfuric acid, ${ }^{10}$ using amorphous carbon and silica sources such as the rice hulls, ${ }^{11}$ using a sol-gel method, ${ }^{12}$ adding ZSM-11 seeds with a shorter crystallization time or using several sources of silicon, ${ }^{13}$ aluminum and alkali metal cations. ${ }^{14}$ Recently, a synthetic route for template-free ZSM-5, which was developed by Yu et al., involved inserting ZSM-11 seeds and decreasing the reaction time from $36 \mathrm{~h}$ to $12-16 \mathrm{~h}$ because the external surface area provided by the seeds accelerated the nucleation rate. Consequently, the small ZSM-5 crystals could be used to achieve high selectivity for $\mathrm{C}_{2}-\mathrm{C}_{4}$ olefins in the methanol to olefins (MTO) reaction. ${ }^{13}$

ZSM-5 has been used as a FCC catalyst since its discovery; however this material has only recently been applied for the pyrolysis of petroleum residues. According to Siddiqui et al., the cracking of Arab Heavy atmospheric residue is affected by the type of catalyst; ZSM-5 disfavors the formation of aromatic carbon and protons in asphaltene while selectively forming linear, low molecular weight hydrocarbons. However, Fesharaki et al. utilized Iranian vacuum residue during two-stage pyrolytic processes over different catalysts. ${ }^{15,16}$ The cracked oil obtained from the first stage was introduced during the second stage, which was a liquid-phase catalytic reaction. Therefore, the AlMCM-41 demonstrated high performance during catalytic production of light olefins relative to the ZSM-5 in its sodic form (Na-ZSM-5).

This study evaluates the synthesis of ZSM-5 produced without an organic template or seeds and compares the physico-chemical properties of the resultant material with a ZSM-5 zeolite synthesized using an organic template. The catalytic activity of zeolites with an MFI structure during the pyrolysis of high molecular weight hydrocarbons, such as atmospheric petroleum residue (ATR), is evaluated.

\section{EXPERIMENTAL}

\section{Synthesis of templated ZSM-5 zeolite}

ZSM-5 zeolite (ZSM-5) was synthesized with an organic template according to the method proposed by Argauer and Landolt with a silicon/aluminum ratio of $25 .{ }^{17}$ This sample was synthesized using 
amorphous silica gel (Acros Organics, 0.035-0.070 mm), sodium hydroxide (Merck), aluminum sulfate hexadecahydrate (Reagen) and tetrapropylammonium bromide (Sigma-Aldrich) as sources for $\mathrm{Si}, \mathrm{Na}$, $\mathrm{Al}$ and the organic template, respectively. To obtain the synthesis gel, two solutions were prepared in water: solution 1 contained silica gel, aluminum sulfate and sodium hydroxide, and solution 2 contained the template. Both solutions were homogenized for 1 hour at room temperature before they were stirred for 2 hours. The synthesis gel can be described using this stoichiometric proportion: 5.3TPABr:7.15 $\mathrm{Na}_{2} \mathrm{O}$ : $1 \mathrm{Al}_{2} \mathrm{O}_{3}: 50 \mathrm{SiO}_{2}: 1000 \mathrm{H}_{2} \mathrm{O}$. Afterwards, the gel was transferred into PTFE (Polytetrafluoroethylene)-lined stainless-steel autoclaves and heated to $423 \mathrm{~K}$ under autogenous pressure and static conditions for 7 days (168 hours). After crystallization, the autoclave was removed from the oven; after being cooled to room temperature, the contents were homogenized via agitation. The resultant solid was separated from the liquid phase by filtration, washed thoroughly with distilled water until the $\mathrm{pH}$ was below 9 and dried at $373 \mathrm{~K}$ for 12 hours.

\section{Synthesis of ZSM-5 zeolite without a template}

To produce the ZSM-5 zeolite without an organic template (ZSM-5-AT), the methods proposed by Cheng et al. and Costa and Araujo were used, with a silicon/aluminum ratio of $25 .^{9,18}$ The starting materials used for synthesis included the following: amorphous silica gel (Acros Organics, 0.035-0.070 mm), sodium hydroxide (Merck), sodium aluminate (Sigma-Aldrich, $\mathrm{Al}_{2} \mathrm{O}_{3} 50-56 \%, \mathrm{Na}_{2} \mathrm{O}$ $40-45 \%)$ and distilled water. The sodium hydroxide and sodium aluminate were dissolved in water with stirring at reflux $(353 \mathrm{~K})$ to produce the synthesis gel. After half an hour, silica gel was added, and the system remained under the same conditions for 6 hours. The synthesis gel can be described using this stoichiometric proportion: $6 \mathrm{Na}_{2} \mathrm{O}: 1 \mathrm{Al}_{2} \mathrm{O}_{3}: 50 \mathrm{SiO}_{2}: 1250 \mathrm{H}_{2} \mathrm{O}$. Afterwards, the gel was transferred into PTFE (Polytetrafluoroethylene)-lined stainless-steel autoclaves and heated to $453 \mathrm{~K}$ under autogenous pressure and static conditions for 27 hours. The subsequent procedures were the same as those mentioned above.

\section{Calcination of ZSM-5 zeolites}

Both synthesized materials were calcined to remove the template and any physisorbed water during a two-step procedure. For the templated zeolite ZSM-5, the sample was first heated at $10 \mathrm{~K} \mathrm{~min}^{-1}$ from room temperature to $823 \mathrm{~K}$ under nitrogen (flow rate of 100 $\mathrm{mL} \min ^{-1}$ ). After the temperature reached $823 \mathrm{~K}$, the system was maintained under these conditions for 2 hours. Next, the flowing $\mathrm{N}_{2}$ was replaced by $100 \mathrm{~mL} \mathrm{~min}^{-1}$ synthetic air at, and the system was maintained under these conditions for 8 hours. The calcination procedure for the template-free zeolite ZSM-5 involved heating from room temperature to $773 \mathrm{~K}$ at $10 \mathrm{~K} \mathrm{~min}^{-1}$ under $100 \mathrm{~mL} \mathrm{~min}^{-1} \mathrm{~N}_{2}$ flow. After the temperature reached $773 \mathrm{~K}$, the conditions were maintained for 2 hours. Subsequently, the nitrogen atmosphere was replaced by synthetic air at the same flow rate, and the system was maintained at this temperature for two hours. This heat treatment was performed under a mild, controlled atmosphere to remove any molecules physisorbed during the directed synthetic process.

\section{Ion exchange in ZSM-5 zeolites}

To generate samples in their protonic form (H-zeolite), all calcined samples were submitted to ion exchange; the $\mathrm{Na}^{+}$cations were replaced by protons $\left(\mathrm{H}^{+}\right)$. The complete ion exchange procedure was performed three times with a $1.0 \mathrm{~mol} / \mathrm{L} \mathrm{NH}_{4} \mathrm{Cl}$ solution at $353 \mathrm{~K}$ for 2 hours. Afterwards, the materials were washed with warm distilled water, filtrated and dried at $373 \mathrm{~K}$ for 12 hours. Finally, all samples were calcined again at $773 \mathrm{~K}$ for 3 hours to generate the protonic form.

\section{Characterization of protonic ZSM-5 zeolites}

The crystalline phases formed after the crystallization process were identifield using X-ray diffraction (XRD) in a SHIMADZU XRD 6000 with Ni-filtered $\mathrm{CuK} \alpha$ radiation $(\lambda=0.1542 \mathrm{~nm})$ and diffraction angles $(2 \theta)$ ranging from $3-40^{\circ}$. The average diameter of the crystallographic domain for each plane (hkl) was calculated using the Scherrer equation : $D_{h k l}=(K . \lambda) /(\beta \cdot \cos \theta)$, where $\mathrm{K}=0.9$ (Constant), $\lambda=$ Wavelength of X-ray source, $\beta=$ full width at half maximum (FWHM) of diffraction, $\theta=$ Bragg angle of the diffraction plane (hkl). ${ }^{19}$ The particle size distribution was assessed using laser granulometry with a particles analyzer Malvern 2000E from MASTERSIZE. Distilled water was used as a dispersion medium for the samples while the particles were deagglomerating in an ultrasonic bath. The chemical compositions were determined using inductively coupled plasma atomic emission spectroscopy (ICP-AES) with a Varian VISTA-AX-CCD. The samples were digested beforehand with $\mathrm{H}_{2} \mathrm{SO}_{4}$ and $\mathrm{HF}$. The $\mathrm{Si} / \mathrm{Al}$ atomic ratios of the samples could be determined through their chemical composition. The morphology of the crystals was determined using scanning electron microscopy (SEM) with a Philips ESEM microscope at 10.000x magnification under vacuum. The functional groups contained in the structures were identified using Fourier Transform Infrared Spectroscopy (FTIR) from 4000 to $400 \mathrm{~cm}^{-1}$ at a $4 \mathrm{~cm}^{-1}$ resolution in a BOMEM MB 102 model spectrophotometer. Argon adsorption-desorption isotherms at $87 \mathrm{~K}$ were also recorded for the zeolite samples using a Quantachrome Autosorb $1 \mathrm{MP}$ automated gas sorption system. Before the measurements, the individual samples were outgassed at $573 \mathrm{~K}$ under vacuum for 16 hours. The surface areas were determined using the Brunauer-Emmet-Teller (BET) equation. The pore size distributions were calculated using the adsorption branch of the argon isotherms via the NL-DFT model (Non Localized Density Functional Theory). The acidic properties of the samples were determined using ammonia temperature programmed desorption (TPD) in a Micromeritics AutoChem 2910 (TPD/TPR) system with $\mathrm{He}$ as the carrier gas. The samples were outgassed in preparation for this process at $833 \mathrm{~K}$ under flowing helium over $30 \mathrm{~min}$. After cooling to $453 \mathrm{~K}$, ammonia flow was passed through the sample for $30 \mathrm{~min}$; subsequently, the physisorbed ammonia was removed using flowing helium at $453 \mathrm{~K}$ over $90 \mathrm{~min}$. The chemisorbed ammonia was measured by increasing the temperature at $15 \mathrm{~K} \mathrm{~min}^{-1}$ up to $823 \mathrm{~K}$ and holding this temperature for $30 \mathrm{~min}$. The ammonia concentration in the effluent helium stream was monitored with a thermal conductivity detector (TCD).

\section{Pyrolysis of atmospheric petroleum residue under the Zeolites ZSM-5}

Atmospheric Petroleum Residue (ATR) was collected at the Industrial Area of Guamaré - PETROBRAS, in Rio Grande do Norte - Brazil. For the pyrolysis process over the ZSM-5 zeolites, a fast pyrolysis method was followed using Single-Shot Pyrolyzer equipment (PY-2020iS model, FRONTIER LAB) with a fixed bed reactor coupled with on-line $\mathrm{CG} / \mathrm{MS}$. The pyrolysis reactions were conducted in quartz filler tubes (62 mm length, $6 \mathrm{~mm}$ i.d.) at 773 K over approximately 3 seconds with $10 \%$ wt of catalyst in ATR, generating $\sim 0,5 \mathrm{mg}$ total mass. The pyrolysis vapors were analyzed using a GC/MS QP2010 Plus model from SHIMADZU. The injector temperature was maintained at $523 \mathrm{~K}$. The chromatographic separation was performed using a UA5-30M-0.25F capillary column (30 m x 0.25 mm i.d., $0.25 \mu \mathrm{m}$ film thickness). Helium (99.999\%) 
was used as the carrier gas at $1.01 \mathrm{~mL} \mathrm{~min}^{-1}$ and with a 1:400 split ratio. The oven temperature was programmed to increase from 313 $\mathrm{K}(2 \mathrm{~min})$ to $573 \mathrm{~K}(10 \mathrm{~min})$ at $10 \mathrm{~K} \mathrm{~min}^{-1}$. The temperature of the GC/MS interface was held at $573 \mathrm{~K}$, and the mass spectrometer was operated in EI mode at $70 \mathrm{~V}$ for ionization, $0.80 \mathrm{kV}$ for the detector and at $553 \mathrm{~K}$ at the ion source. The mass spectra were obtained from $\mathrm{m} / \mathrm{z} 29$ to 600 at $588 \mathrm{amu} / \mathrm{s}$. The results are reported as the average of two runs for each experiment, and the chromatographic peaks were identified using the NIST (National Institute of Standards and Technology) MS library.

\section{RESULTS AND DISCUSSION}

The X-ray diffraction data were analyzed to identify the phase of the microporous zeolites and to evaluate the crystallinity of the obtained materials. The X-ray diffraction patterns for the ZSM-5 zeolites synthesized with and without templates, and a commercial sample are shown in Figure 1. For both ZSM-5 zeolites synthesized, an MFI crystalline structure formed, according to the database of the Joint Committee on Powder Diffraction Standards (JCPDS, COD. 49-0657) and the International Zeolite Association (IZA). The high intensity of the peaks in the diffractograms for both ZSM-5 indicated significant crystallinity as compared to commercial sample (Table 1), even after the thermal processes used to obtain the protonated zeolites according to literature. , $^{4,20}$

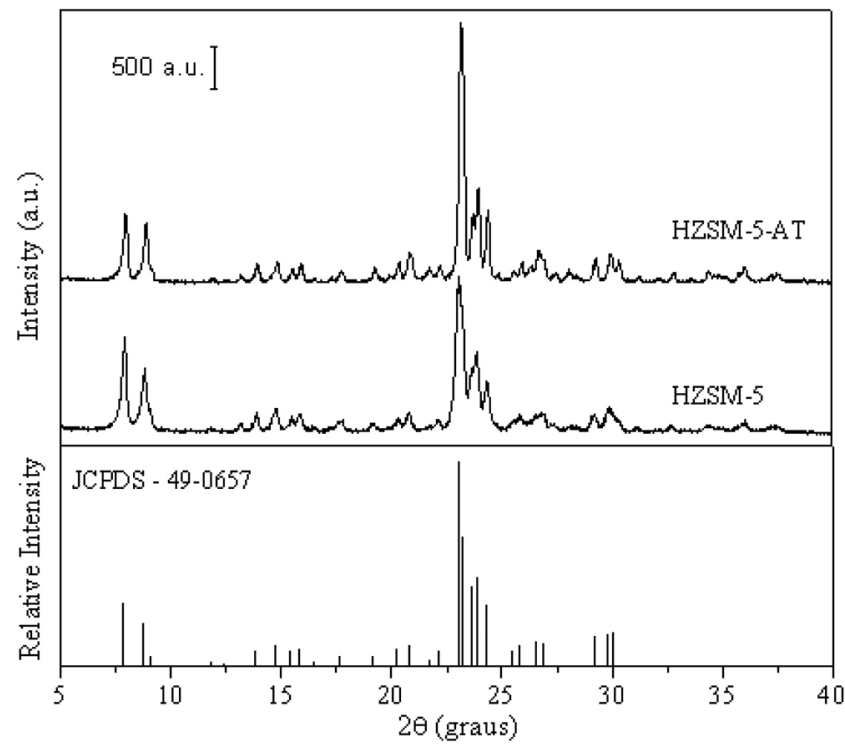

Figure 1. X-Ray diffractograms of protonic ZSM-5 zeolites synthesized, commercial ZSM-5 and standard JCPDS

In Figure 2, the FTIR absorption spectra for the protonated zeolites from 4000 to $400 \mathrm{~cm}^{-1}$ are presented. All of the zeolites displayed a large, intense band near the $3445 \mathrm{~cm}^{-1}$, which is assigned to siloxane and/or hydroxyl groups formed by water molecules physisorbed to the surface. The band at $1635 \mathrm{~cm}^{-1}$ corresponds to the deformational vibrations of water molecules. The bands at 1220 and $1097 \mathrm{~cm}^{-1}$ represent the external and internal asymmetric stretching of the siloxane groups. The band at $792 \mathrm{~cm}^{-1}$ represents the symmetric stretching of the siloxane groups. The internal flexions of the tetrahedrons correspond to the band at $543 \mathrm{~cm}^{-1}$. The band at $450 \mathrm{~cm}^{-1}$ is attributed to the presence of five-membered rings.

Therefore, it was possible to prove that the synthetic conditions employed to produce the ZSM-5 without a template were proven efficient by evaluating the crystal structure using XRD and FTIR; only the ZSM-5 zeolite phase was observed, in accordance with the literature. ${ }^{9,21,22}$

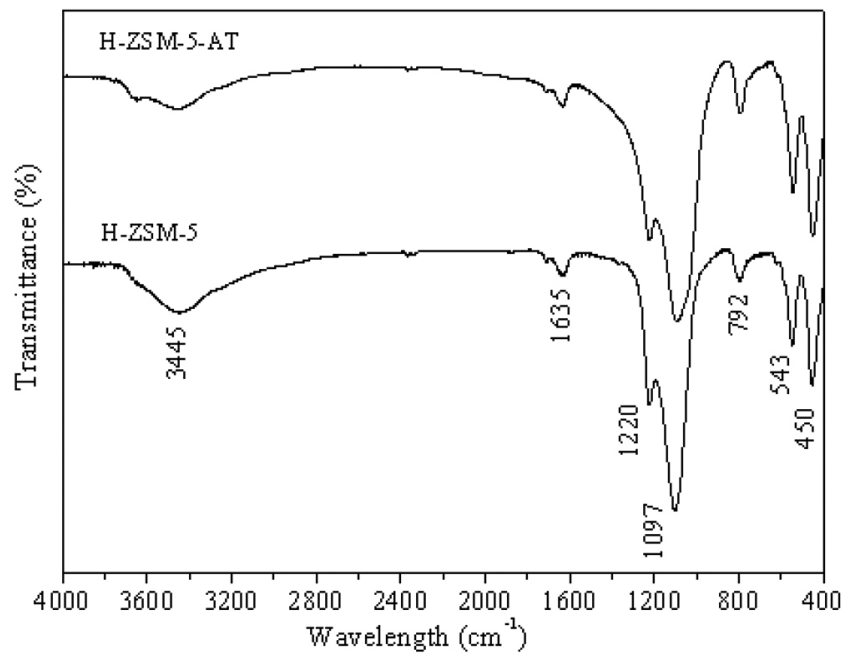

Figure 2. FTIR of the ZSM-5 zeolites in protonic form

The micrographs of the zeolites revealed orthorhombic morphologies, as shown in Figure 3 (A-B). The HZSM-5-AT forms as crystallites with very well defined prismatic shapes, according to Narayanan, et al, while ZSM-5 forms as an agglomerate of small crystals. $^{7}$ The HZSM-5-AT exhibited a larger particle diameter relative to the HZSM-5, as observed in the graph presenting the particle size distribution (Figure 3-C). As indicated in Table 1, the values calculated using the Scherrer equation for the crystallite diameters $\left(D_{h k l}\right)$ correspond to the average value for all of the planes identified in each zeolite sample (according to XRD analysis). The template-free ZSM-5 exhibited larger crystallite diameters than the ZSM-5 synthesized with a template; this result agrees with the XRD diffractograms because the ZSM-5-AT produced diffractogram peaks with a lower full width at half maximum (FWHM) due to the higher intensity of its peaks relative to ZSM-5. According to the Scherrer equation, the FWHM of each plane (hkl) is inversely proportional to the crystallite diameters. The crystallites diameter $\left(D_{\mathrm{hkl}}\right)$ influences the particle size; therefore, the ZSM-5-AT has a larger particle size, as shown in Figure 3. After graphing the particle size distribution (Figure 3), the $\mathrm{d}(0.5)$ parameter could be calculated; this value divides the distribution in half. This parameter $d(0.5)$ is reported in Table 1 and shows that the value for the ZSM-5-AT was almost double that of the HZSM-5, as demonstrated in the micrographs of both zeolites.

After analyzing the chemical composition by ICP-AES (Table 1), the concentration of silicon and aluminum could be determined for samples of both zeolites. According to these concentrations, the real values for the $\mathrm{Si} / \mathrm{Al}$ molar ratio were lower than the theoretically calculated value, but the values are similar between the zeolites. These results agree with those obtained by Sang et al., who achieved $85 \%$ crystallinity while synthesizing template-free ZSM-5 with a Si/Al molar ratio of 25 . Kim et al. organized a crystallization field for the template-free ZSM-5; for the molar ratios of $\mathrm{SiO}_{2} / \mathrm{Al}_{2} \mathrm{O}_{3}=50$ and $\mathrm{Na}_{2} \mathrm{O} / \mathrm{SiO}_{2}=0.12$, only the ZSM-5 phase forms, corroborating the results in this work. ${ }^{4,8}$

To evaluate the textural properties of the zeolites, specifically the microporosity, argon physisorption was carried out at $87.3 \mathrm{~K}$ (Figure 4). According to the pore size distribution (dV/dlogDp) obtained using the NL-DFT method, the first peak has a maximum close to $5.5 \mathrm{~A}$ and corresponds to the diameter of the characteristic micropores of ZSM-5. However, the second peak in the ranges between 7-11 A does not correspond to a relevant pore distribution, so 


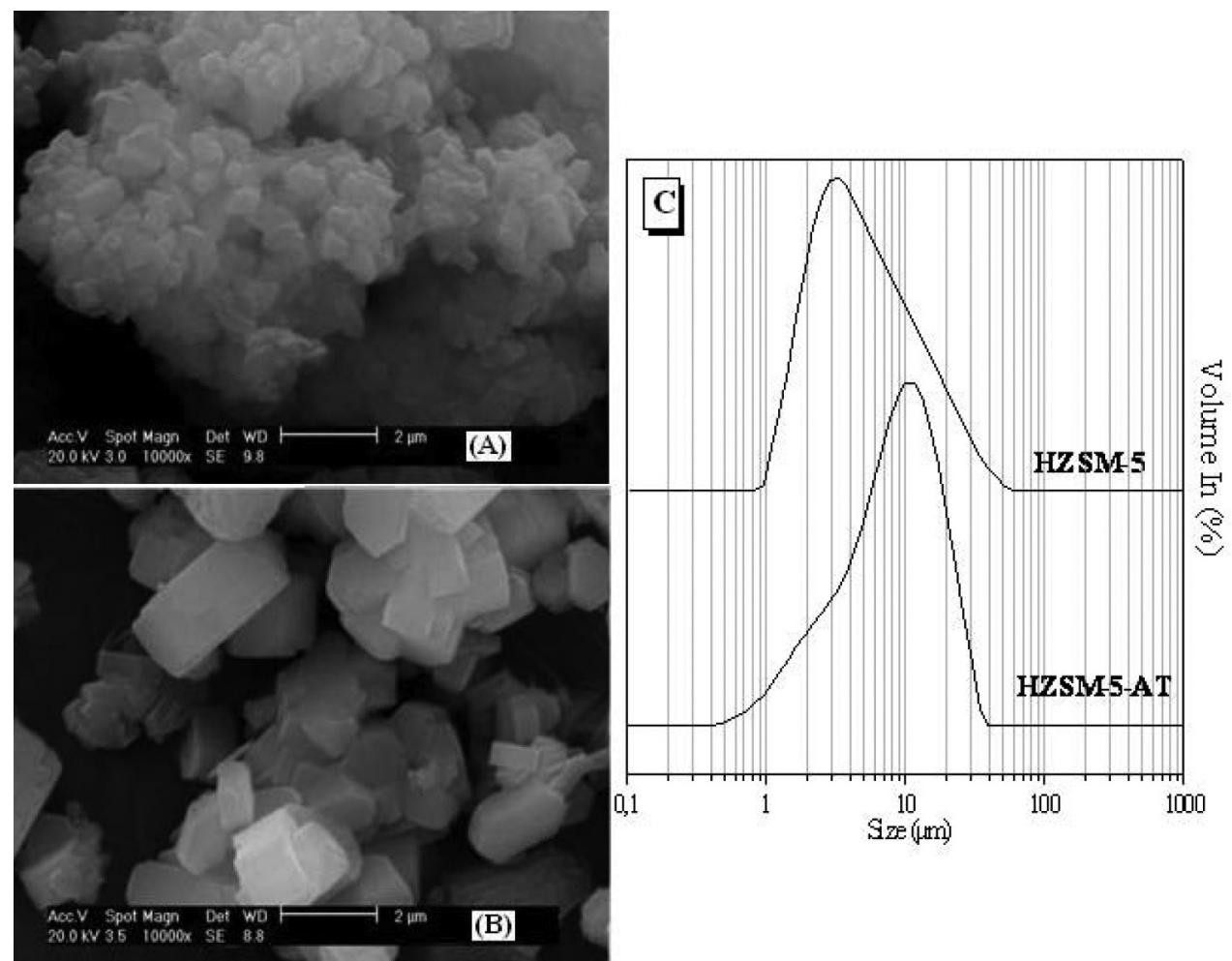

Figure 3. Micrographs of zeolites HZSM-5(A) and HZSM-5-AT (B) in protonic form. Curves describing the particle size distribution for both zeolites $(C)$

Table 1.Textural properties, crystallographic parameters and chemical composition of the ZSM-5 zeolites in protonic form

\begin{tabular}{|c|c|c|}
\hline \multirow{2}{*}{ Properties } & \multicolumn{2}{|c|}{ Samples } \\
\hline & HZSM-5 & HZSM-5-AT \\
\hline Relative Crystallinity $(\%)^{\mathrm{a}}$ & 87 & 83 \\
\hline Specific Area $\left(\mathrm{m}^{2} \mathrm{~g}^{-1}\right)^{\mathrm{b}}$ & 374 & 301 \\
\hline Micropore Area $\left(\mathrm{m}^{2} \mathrm{~g}^{-1}\right)^{\mathrm{c}}$ & 300 & 257 \\
\hline External Area $\left(\mathrm{m}^{2} \mathrm{~g}^{-1}\right)^{\mathrm{c}}$ & 74 & 44 \\
\hline Micropore volume $\left(\mathrm{cm}^{3} \mathrm{~g}^{-1}\right)^{\mathrm{c}}$ & 0.187 & 0.160 \\
\hline External volume $\left(\mathrm{cm}^{3} \mathrm{~g}^{-1}\right)^{\mathrm{c}}$ & 0.111 & 0.042 \\
\hline $\mathrm{D}_{\mathrm{hkl}}(\mathrm{nm})^{\mathrm{d}}$ & 25.66 & 42.74 \\
\hline $\mathrm{d}(0.5)(\mu \mathrm{m})^{\mathrm{e}}$ & 4.875 & 9.151 \\
\hline $\mathrm{C}_{\mathrm{Al}}(\% \mathrm{wt})^{\mathrm{f}}$ & 2.20 & 2.41 \\
\hline Ratio $\mathrm{Si} / \mathrm{Al}^{\mathrm{f}}$ & 19 & 18 \\
\hline $\mathrm{T}_{\text {máx }}(\mathrm{K})^{\mathrm{g}}$ & 613 & 640 \\
\hline Acidity $\left(\text { meqNH}_{3} \mathrm{~g}^{-1}\right)^{\mathrm{g}}$ & 0.399 & 0.439 \\
\hline
\end{tabular}

$\mathrm{a}-$ Calculated using commercial ZSM-5 as standard; $\mathrm{b}$ - Calculated using the B.E.T. method; $\mathrm{c}-$ Calculated using the NL-DFT method; $\mathrm{d}-$ Calculated using the Scherrer equation; e - Particles analyzer Malvern 2000E; f - Determined by ICP-AES.; $\mathrm{g}$ - Determined by TPD.

these phenomena are associated with the adsorbent and adsorbate. According to the literature,${ }^{23}$ these phenomena may be associated with fluid-to-crystalline-like phase transitions of the adsorbate on the MFI micropore. However, according to Garcia-Perez et al., computer simulations using flexible MFI frameworks suggests a monoclinic to orthorhombic transition. ${ }^{24}$ The values for the textural properties calculated using the NL-DFT method are shown in Table 1. As revealed by the cumulative pore volume, the templated ZSM- 5 exhibited slightly higher micropore volumes (5.5 A), but the microporosity of the zeolites was not affected by the average particle diameter. Both samples presented specific areas appropriate for ZSM-5. However, HZSM-5 presented a large external area and volume. These results are related to the average particle diameter because smaller particles allow greater accessibility and generate a larger contact surface. Consequently, the agglomeration of these particles, which present smaller average particle diameters generates an interstitial porosity represented by an increase in the pore volume from 50-900 A for HZSM-5, as observed in Figure 4.

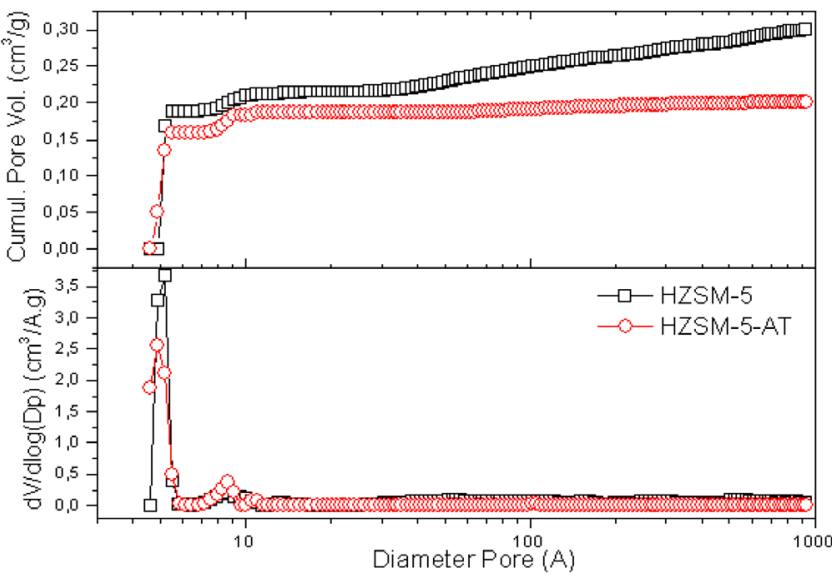

Figure 4. Pore size distribution and cumulative pore volume obtained from NL-DFT calculations of Ar adsorption-desorption at $87 \mathrm{~K}$

Thermal ammonia desorption is an effective technique characterizing the acidic properties of the zeolites because it provides information about the total number of acid sites (calculated from the $\mathrm{NH}_{3}$ detected during desorption) and their acidic strength (as related to the temperature of the peak maximum in the desorption curve). Figure 5 shows the thermal desorption of $\mathrm{NH}_{3}$ for ZSM- 5 zeolites synthesized with and without templates; the acidic properties of the 
zeolites could be calculated from these curves (Table 1). In both of the ZSM-5 samples, the number of acid sites was inversely proportional to the $\mathrm{Si} / \mathrm{Al}$ molar ratio, indicating that all of the aluminum sites were accessible to the ammonia molecules. However, the template-free ZSM-5 contained additional acid sites due to its aluminum content. The total acidity for both zeolites matches that reported in the literature; Xue et al. obtained similar values $\left(0.46 \mathrm{mmol} \mathrm{NH}_{3} \mathrm{~g}^{-1}\right)$ for nano-sized, protonated ZSM-5 with equivalent $\mathrm{Si} / \mathrm{Al}$ ratios. ${ }^{25} \mathrm{In}$ addition, the maximal temperatures for ammonia desorption indicates the strength of the acid sites; and HZSM-5-AT exhibited higher temperatures $(640 \mathrm{~K})$ than the HZSM-5 $(613 \mathrm{~K})$. A high maximum temperature might indicate the formation of additional Brönsted acid sites because the interactions with Brönsted acids are stronger than those with Lewis acids. Therefore, the acidic properties of these materials should be investigated using the adsorption-desorption of other bases to distinguish and to isolate the contributions of the Brönsted and Lewis acid sites.

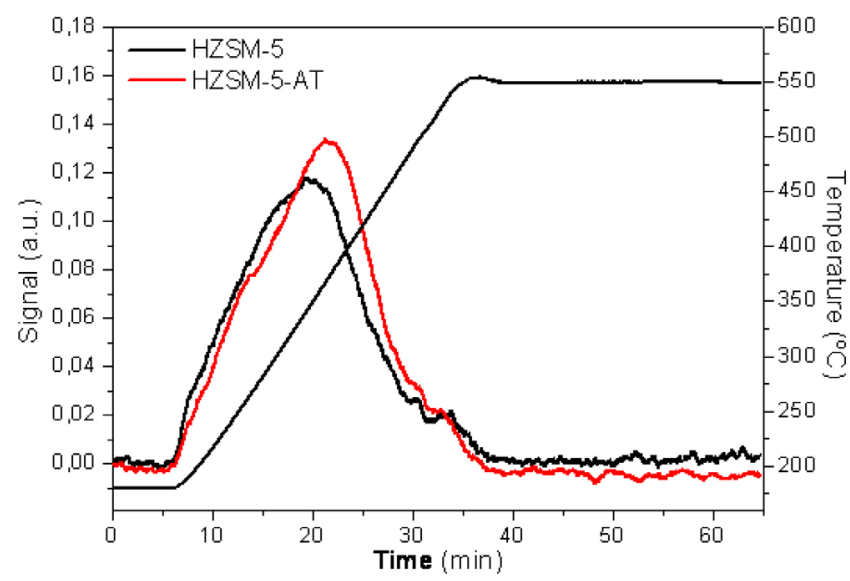

Figure 5. $\mathrm{NH}_{3}$ thermal desorption of calcined zeolites

The fast pyrolysis technique produces vapors that can be analyzed directly using GC/MS. According to Qiang et al.,${ }^{26}$ pyrolysis vapors are not true vapors; rather, they are mixtures of volatile compounds and non-volatile oligomers; GC/MS can only analyze the volatile compounds in the pyrolysis vapors. The temperature was $773 \mathrm{~K}$, selected according to a study by Castro et al.; at this temperature, the atmospheric petroleum residue (ATR) degrades more than $90 \mathrm{wt} \% .^{27}$

Table 2 separates the selectivity values according to the groups of hydrocarbons and selectivity for aromatics produced during the catalytic pyrolysis over zeolite ZSM-5. The ATR is largely composed of high molecular weight compounds; consequently, the thermal pyrolysis of this material produces approximately $95 \%$ of hydrocarbons in band 13 to 32, which is classified as gas-oil or diesel oil. When performing the catalytic pyrolysis over ZSM-5 zeolites, the production of gas-oil compounds decreases by approximately 20 to $25 \%$, generating light gas products $\left(\mathrm{C}_{2}-\mathrm{C}_{6}\right)$ and 4 to $6 \%$ more compounds in the gasoline band $\left(\mathrm{C}_{7}-\mathrm{C}_{12}\right)$. The formation of light gases has been reported the literature; Fesharaki et al. produced $24.49 \%$ light olefins during the pyrolysis of vacuum residue over Na-ZSM-5. ${ }^{16}$ According to Siddiqui et al., several reactions and mechanisms are possible when following the pyrolytic reactions of residues. Some examples of these reactions might include condensation with ester formation, polymerization or isomerization, dehydrogenation, aromatization, or dealkylation. ${ }^{15}$ The processes necessary for catalytic degradation involves the reaction of a proton from a Brönsted acid site with an ionic mechanism that proceeds along two possible pathways: end-chain scission and random cleavage at any bond in the chain. When comparing the two zeolites, the greater selectivity exhibited by templated ZSM-5 was attributed to its higher external area and smaller particle size distribution (see Table 1); these values indicate additional contact surfaces are available for the diffusion of molecules and mass transfer in the active sites. Even the template-free ZSM-5 presented additional acid sites, as calculated from the $\mathrm{NH}_{3}$ desorption data; the diffusion of probe molecules with small kinetic diameters is larger than that of long carbon chains.

The selectivity values for the aromatic compounds are listed in Table 2; these values were similar for both ZSM-5 zeolites. Specifically, aromatization is catalyzed by Brönsted acid sites during the hydrogen transfer in paraffins and cycloalkanes, generating intermediate compounds such as olefins. ${ }^{28}$ The low selectivity for aromatic compounds agrees with the literature data; Lukyanov observed that HZSM-5 had a decreased ability to catalyze hydrogen transfer reactions that proceed through a bimolecular transition state while using sterically encumbered materials. ${ }^{29}$

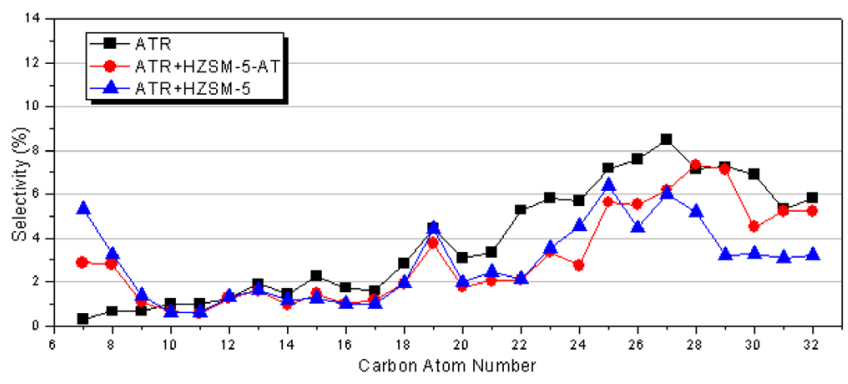

Figure 6. Distribution by carbon atom number obtained during the pyrolysis of ATR over ZSM-5 samples

To aid in understanding the hydrocarbon selectivity during pyrolysis, Figure 6 shows the selectivities for each carbon atom number. Evaluating the compounds produced in the gasoline range $\left(\mathrm{C}_{7}-\mathrm{C}_{12}\right.$ group) revealed that a contribution from 4 to $7 \%$ during the catalytic pyrolysis was provided only for $\mathrm{C}_{7}$ and $\mathrm{C}_{8}$ hydrocarbons; most of that percentage was attributed to the production of aromatic compounds. Table 2 shows the selectivity of aromatic compounds, where the template-free ZSM-5 presented exactly similar values to the conventional ZSM-5. In addtion, the 20 to $25 \%$ of hydrocarbons produced (Table 2) smaller than $\mathrm{C}_{7}$ was attributed to the conversion of $\mathrm{C}_{32}$ to $\mathrm{C}_{22}$ compounds through an end-chain cracking mechanism. The template-free ZSM-5 produced $20 \%$ of light hydrocarbons $\left(\mathrm{C}_{4^{-}}\right.$ $\mathrm{C}_{6}$ ), where such compounds are olefins and paraffins of great interest to the petrochemical industry.

Table 2. Selectivity data obtained during the pyrolysis of ATR over ZSM-5 zeolites

\begin{tabular}{|c|c|c|c|c|c|c|c|}
\hline \multirow{2}{*}{ SAMPLES } & \multicolumn{3}{|c|}{ Selectivity per Carbon Group (\%) } & \multicolumn{4}{|c|}{ Selectivity by Aromatics (\%) } \\
\hline & $\mathrm{C}_{4}-\mathrm{C}_{6}$ & $\mathrm{C}_{7}-\mathrm{C}_{12}$ & $\mathrm{C}_{13}-\mathrm{C}_{32}$ & Toluene & Ethyl-Benzene & Xylenes & Dimetyl-Naphthalene \\
\hline ATR & 0 & 4.83 & 95.17 & 0 & 0 & 0 & 0 \\
\hline ATR+HZSM-5 & 25.48 & 12.49 & 62.03 & 1.83 & 0.45 & 1.92 & 0.70 \\
\hline ATR+HZSM-5-AT & 20.17 & 9.21 & 70.62 & 1.88 & 0.37 & 1.97 & 0.67 \\
\hline
\end{tabular}




\section{CONCLUSIONS}

The synthesis procedure for obtaining the ZSM-5 zeolite without an organic template was effective because a networked MFI structure formed according to its crystallinity and the microporous structure. In addition, the microporous structure was not destroyed after the heat treatment and ion exchange. The major physico-chemical properties of template-free ZSM-5 were similar to those of the templated ZSM-5, diverging only in average particle diameter and external surface area. However, these differences can be reduced by altering the synthetic parameters. In addition, a template-free ZSM-5 could be produced when the synthesis time was reduced from 7 days (conventional synthesis) to only 27 hours after modifying the crystallization temperature in $30 \mathrm{~K}$ (conventional $-423 \mathrm{~K}$; to template-free $-453 \mathrm{~K}$ ). The procedure for synthesizing template-free ZSM-5 facilitated the incorporation of aluminum atoms into the crystal lattice, generating stronger acid sites that may indicate the formation of Brönsted acid sites.

During the pyrolysis of petroleum atmospheric residue (ATR), both zeolites showed catalytic activity when cracking high molecular weight hydrocarbons. The templated ZSM-5 zeolite was more active toward producing light compounds due to its larger contact surface. However, the template-free ZSM-5 produced $20 \%$ of light compounds, a significant percentage for transform atmospheric petroleum residue to paraffins and olefins, for the petrochemical industry. In addition, both zeolites showed similar capacities for hydrogen transfer during aromatization reactions, making the template-free ZSM-5 as efficient as the conventional ZSM-5 independent of the external surface area.

The acidic template-free ZSM-5 zeolite is promising for use as an industrial catalyst due to its low production costs, shorter synthesis times and better density and positioning for the acid centers compared to templated ZSM-5 zeolite.

\section{ACKNOWLEDGEMENTS}

The authors are immensely grateful to Professor Dr. David P. Serrano at the "Grupo de Ingeniería Química y Ambiental" of the "Universidad Rey Juan Carlos" (URJC, Spain) for performing the ICP-AES, TPD of $\mathrm{NH}_{3}$ and Argon adsorption-desorption analyses. The authors also thank the "Coordenação de Apoio a Pessoal de Ensino Superior" (CAPES, Brazil) for financial support.

\section{REFERENCES}

1. Pace, G. G.; Rendón, A. M.; Fuentes, G. R.; Zeolitas: características, propiedades y aplicaciones industriales. Ed. Innovación Tecnológica: Caracas, 2000, p. 351

2. Braga, A. A. C.; Morgon, N. H.; Quim. Nova 2007, 30, 178.

3. Kim, S. D.; Noh, S. H.; Park, J. W.; Kim, W. J.; Microporous Mesoporous Mater. 2006, 92, 181.
4. Sang, S.; Chang, F.; Liu, Z.; He, C.; He, Y.; Xu, L.; Catal. Today 2004, 93, 729.

5. Mignoni, M. L.; Detoni, C.; Pergher, S. B. C.; Quim. Nova 2007, 30, 45.

6. Materiales zeolíticos: síntesis, propiedades y aplicaciones; Martínez, J. G.; Pariente, J. P., eds.; Publicaciones Universidad de Alicante: Alicante, 2002, p. 210.

7. Narayanana, S.; Sultana, A.; Le, Q. T; Auroux, A.; Appl. Catal., A 1998, $168,373$.

8. Kim, S. D.; Noh, S. H.; Seong, K. H.; Kim, W. J.; Microporous Mesoporous Mater. 2004, 72, 185.

9. Cheng, Y.; Liao, R. H.; Li, J. S.; Sun, X. Y.; Wang, L. J. J.; Mater. Process. Technol. 2008, 206, 445.

10. Kang, N. Y.; Song, B. S.; Lee, C. W.; Choi, W. C.; Yoon, K. B.; Park, Y. K.; Microporous Mesoporous Mater. 2009, 118, 361.

11. Vempati, R. K.; Borade, R.; Hegde, R. S.; Komarneni, S.; Microporous Mesoporous Mater. 2006, 93, 134.

12. Han, W.; Jia Y.; Xiong, G.; Yang, W.; Sci. Technol. Adv. Mater. 2007, 8101,105

13. Yu, Q.; Meng, X.; Liu, J.; Li, C.; Cui, Q.; Microporous Mesoporous Mater. 2013, 181, 192.

14. Zhang, L.; Liu, S.; Xie, S.; Xu, L.; Microporous Mesoporous Mater. 2012, 147, 117 .

15. Siddiqui, M. N.; J. Anal. Appl. Pyrolysis 2010, 89, 278.

16. Fesharaki, M. J.; Ghashghaee, M.; Karimzadeh, R.; J. Anal. Appl. Pyrolysis 2013, 102, 97.

17. Argauer, R. J.; Landolt, G. R.; US Pat. RE 298571978

18. Costa, M. J. F.; Araujo, A. S.; Revista da Propriedade Industrial 2010, $2083,76$.

19. Zhan, B. Z.; White, M. A.; Lumsden, M.; Mueller-Neuhaus, J.; Robertson, K. N.; Cameron, T. S.; Gharghouri, M.; Chem. Mater 2002, 14, 3636.

20. Woolery, G. L.; Kuehl, G. H.; Timken, H. C.; Chester, A. W.; Vartuli, J. C.; Zeolites 1997, 19, 288.

21. Aguado, J.; Serrano, D. P.; Escola, J. M.; Rodríguez, J. M.; Microporous Mesoporous Mater. 2004, 75, 41.

22. Burkett, S. L.; Davis, M. E.; J. Phys. Chem. 1994, 98, 4647.

23. Groen, J. C.; Peffer, L. A. A.; Pérez-Ramírez, J.; Microporous Mesoporous Mater. 2003, 60, 1.

24. García-Pérez, E.; Parra, J. B.; Ania, C. O.; Dubbeldam, D.; Vlugt, T. J. H.; Castillo, J. M.; Merkling, P. J.; Calero, S.; J. Phys. Chem. C 2008, $112,9976$.

25. Xue, T.; Wang, Y. M.; He, M. Y.; Microporous Mesoporous Mater. 2012, $156,29$.

26. Qiang, L.; Wen-zhi, L.; Dong, Z.; Xi-feng, Z.; J. Anal. Appl. Pyrolysis 2009, 84, 131 .

27. Castro, K. K. V.; Paulino, A. A. D.; Silva, E. F. B.; Chellappa, T.; Lago, M. B. D. L.; Fernandes Jr, V. J.; Araujo, A. S.; J. Therm. Anal. Calorim. 2011, 106, 759

28. Corma, A.; Faraldos, M.; Martínez, A.; Mifsud, A.; J. Catal. 1990, 122, 230 .

29. Lukyanov, D. B.; J. Catal. 1994, 145, 54. 